Supporting information

\title{
Redispersion strategies for dried cellulose
}

\section{nanofibrils}

Malin Nordenström ${ }^{a, b, \dagger}, *$, Tahani Kaldéus ${ }^{a, b \dagger}$, Johan Erlandsson $^{a}$, Torbjörn Pettersson $^{a, b}$, Eva Malmström ${ }^{a, b}$, Lars Wågberg ${ }^{a, b, *}$

a Department of Fibre and Polymer Technology, KTH Royal Institute of Technology,

Teknikringen 56, SE-100 44 Stockholm, Sweden

${ }^{\text {b} W a l l e n b e r g ~ W o o d ~ S c i e n c e ~ C e n t e r, ~ K T H ~ R o y a l ~ I n s t i t u t e ~ o f ~ T e c h n o l o g y, ~ T e k n i k r i n g e n ~ 56, ~ S E-~}$

10044 Stockholm, Sweden

$\uparrow$ These authors contributed equally.

Number of pages: 7

Number of figures: 5

Number of tables: 1 


\section{Long-term colloidal stability}

The colloidal stability of redispersed freeze-dried CNF dispersions was optically evaluated 12 months after the samples were redispersed. Figure S1 shows sedimentation of FD-CNF600-RD, and haziness of FD-CNF600:PEG-RD and FD-CNF700-RD. The other samples (FDCNF700:PEG-RD, FD-CNF1200-RD and FD-CNF1200:PEG-RD) showed no signs of colloidal instability after 12 months.
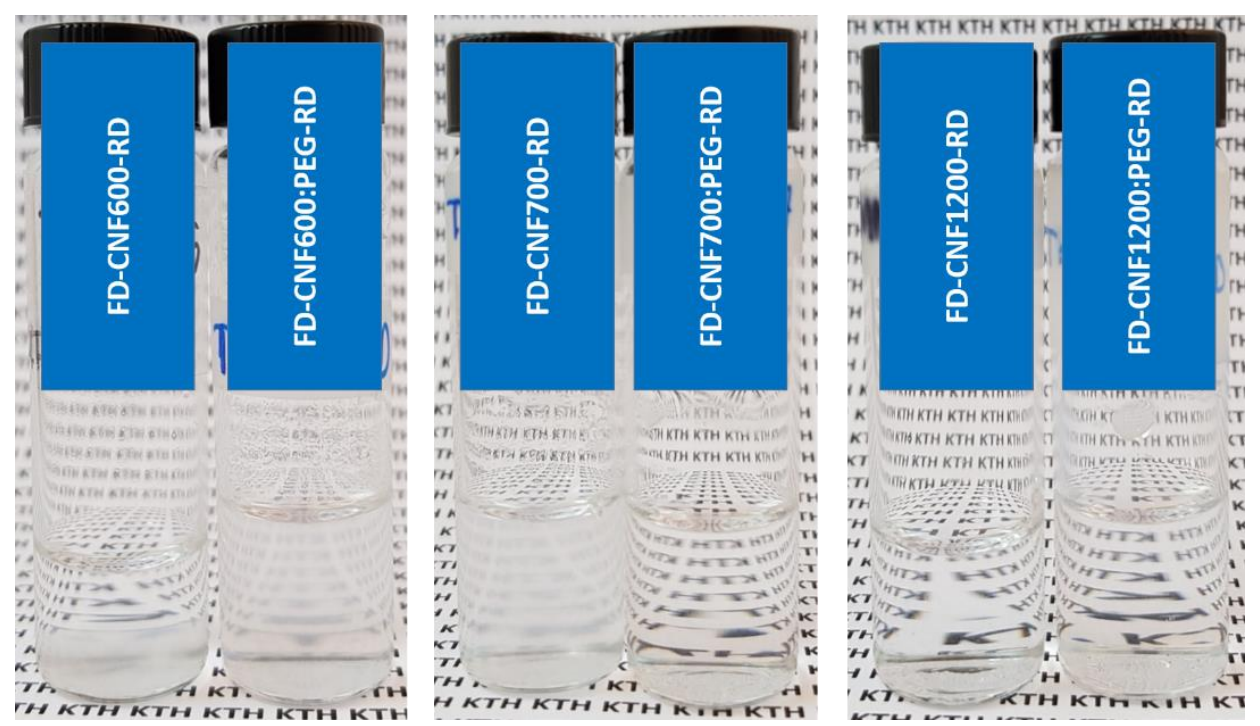

Figure S1. Long-term stability, after storing for 12 months, of redispersed freeze-dried CNF of different surface charge densities, with and without $20 \mathrm{wt} \%$ PEG.

\section{High-resolution scanning electron microscopy (SEM)}

Images showing the morphology of freeze-dried dispersions and nanopapers were obtained with an S-4800 field emission scanning electron microscope (SEM) (Hitachi, Tokyo, Japan). Both the nanopapers and freeze-dried dispersions were imaged with a working distance of $8 \mathrm{~mm}$ using an accelerating voltage of $1 \mathrm{kV}$. The surface morphology of the cross-sections of the nanopapers was studied on samples mounted flat on a conducting carbon tape and the interior morphology was studied by first freeze-fracturing the nanopapers and subsequently imaging the cross-section using 
a split-mount sample holder. The freeze-dried dispersions were carefully cut into thin pieces and mounted flat on the conducting carbon tape. Prior to imaging, both the freeze-dried dispersions and nanopapers were sputter coated with Pt/Pd-alloy using a Cressington 208 HR (Cressington Scientific Instruments, Watford, UK) for $20 \mathrm{~s}$ to limit charging of the specimen during imaging.

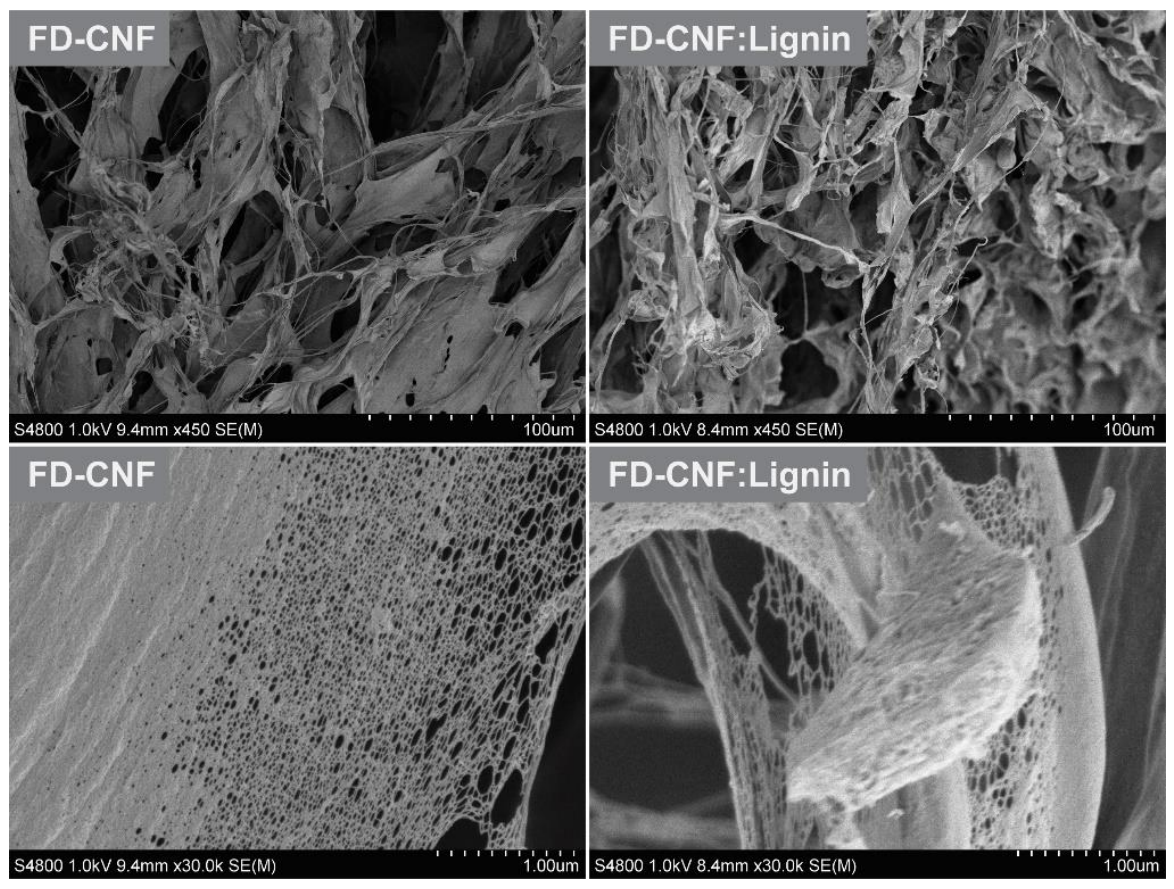

Figure S2. SEM images of freeze-dried CNF dispersions, without (left) and with $10 \mathrm{wt} \%$ lignin (right). 

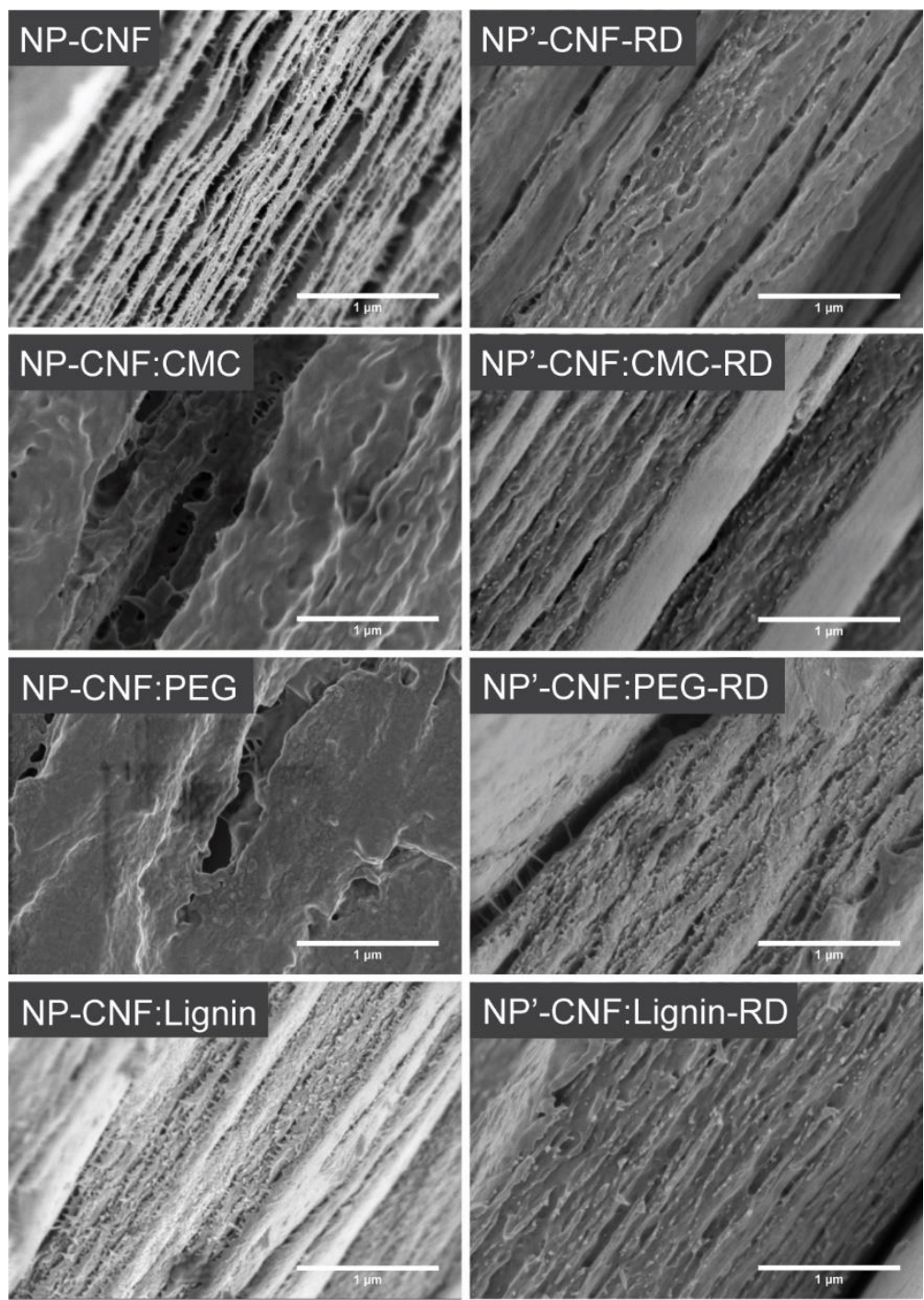

Figure S3. SEM-images of the cross-sections of the nanopapers prepared from never-dried (NPCNF) or redispersed CNF (NP'-CNF-RD), showing the layered structure of the CNFs formed during filtration. The scale bars are $1 \mu \mathrm{m}$. 

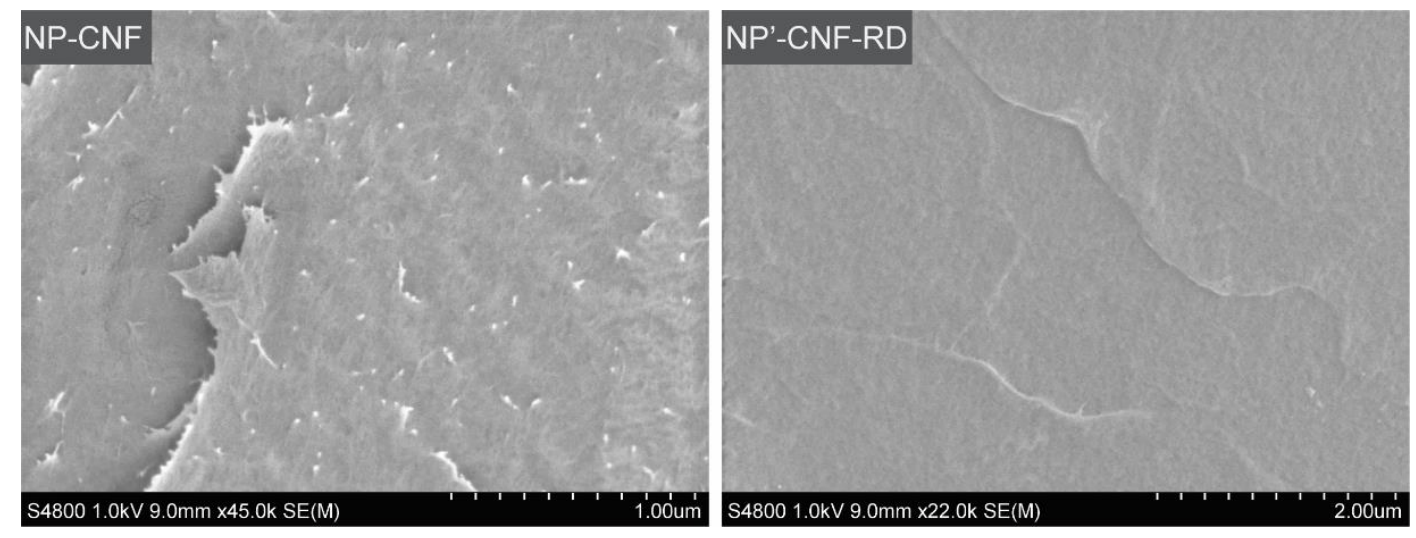

Figure S4. SEM-images of the surfaces of the nanopapers prepared from never-dried (NP-CNF) or redispersed CNF (NP'-CNF-RD).

\section{AFM imaging of lignin particles}

AFM imaging (Multimode 8, Bruker, USA) of a lignin-coated surface was carried with a cantilever with a resonance frequency of $150 \mathrm{kHz}$, a spring constant of $5 \mathrm{Nm}^{-1}$, and a tip radius of $8 \mathrm{~nm}$ (RTESP150, Bruker, US). The surface was prepared by immersing a freshly cleaved mica sheets in PEI $\left(0.1 \mathrm{~g} \mathrm{~L}^{-1}, 10 \mathrm{~s}\right)$, Milli-Q water, lignin dispersion $\left(0.1 \mathrm{~g} \mathrm{~L}^{-1}, 10 \mathrm{~s}\right)$ and Milli-Q water, followed by drying with nitrogen gas.

The AFM image (Figure S5) shows small colloidal lignin particles, as well as larger structures. One example of such a large structure is indicated by the white arrow. 

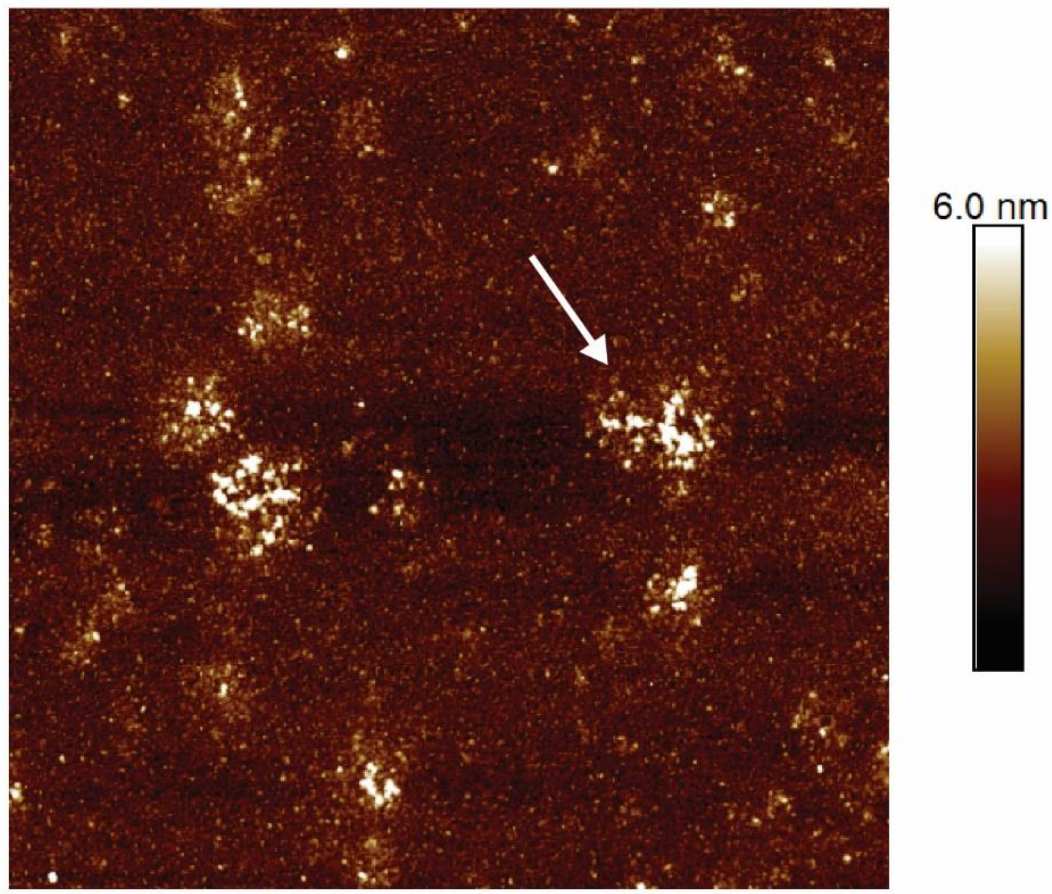

Figure S5. AFM height image of lignin particles, obtained in the SCAN ASYST-AIR mode. The image size is $2 \times 2 \mu \mathrm{m}$. The arrow indicates one of the large structures formed by the lignin particles.

\section{UV-vis spectrophotometry}

The optical properties of CNF nanopapers were studied using a Shimadzu UV-2550 spectrophotometer combined with an integrating sphere. For each nanopaper the total and diffuse transmittance was measured at three different positions. The haze was calculated as Haze $(\%)=$ (diffuse transmittance/total transmittance) $\times 100$.

The total transmittance (Table S1) was the same for the all samples, except NP'-CNF:Lignin$\mathrm{RD}$ for which the transmittance was lower due to the brown color of the sample. The haze of the nanopapers prepared from redispersed CNF was similar to that of the nanopaper made from neverdried CNF, even without the addition of redispersing agent. In fact, the haze of NP'-CNF-RD was slightly lower than that of the other samples, probably because the sample was thinner $(35 \mu \mathrm{m}$ compared with $40-50 \mu \mathrm{m})$. 
Table S1. Optical properties and a $95 \%$ confidence interval of nanopapers prepared from redispersed or never-dried $\mathrm{CNF}$

\begin{tabular}{lll}
\hline Sample & Total transmittance $(\%)$ & Haze $(\%)$ \\
\hline NP-CNF & $86.7 \pm 2.3$ & $19.1 \pm 2.1$ \\
NP'-CNF-RD & $87.2 \pm 0.4$ & $13.3 \pm 2.3$ \\
NP'-CNF:CMC-RD & $88.1 \pm 0.9$ & $18.1 \pm 2.1$ \\
NP'-CNF:PEG-RD & $87.3 \pm 0.7$ & $16.2 \pm 0.8$ \\
NP'-CNF:Lignin-RD & $79.8 \pm 0.3$ & $19.8 \pm 2.3$ \\
\hline
\end{tabular}

